\title{
A Bayesian Approach for Liver Analysis: Algorithm and Validation Study
}

\author{
M. Freiman ${ }^{1}$, O. Eliassaf ${ }^{1}$, Y. Taieb ${ }^{1}$, L. Joskowicz ${ }^{1}$, and J. Sosna ${ }^{2}$ \\ ${ }^{1}$ School of Eng. and Computer Science, The Hebrew Univ. of Jerusalem, Israel \\ ${ }^{2}$ Dept. of Radiology, School of Medicine, Hadassah Hebrew Univ. Medical Center, \\ Jerusalem, Israel \\ freiman@cs.huji.ac.il
}

\begin{abstract}
We present a new method for the simultaneous, nearly automatic segmentation of liver contours, vessels, and metastatic lesions from abdominal CTA scans. The method repeatedly applies multi-resolution, multi-class smoothed Bayesian classification followed by morphological adjustment and active contours refinement. It uses multi-class and voxel neighborhood information to compute an accurate intensity distribution function for each class. The method requires only one or two user-defined voxel seeds, with no manual adjustment of internal parameters. A retrospective study on two validated clinical datasets totaling 56 CTAs was performed. We obtained correlations of 0.98 and 0.99 with a manual ground truth liver volume estimation for the first and second databases, and a total score of 67.87 for the second database. These results suggest that our method is accurate, efficient, and robust to seed selection compared to manually generated ground truth segmentation and to other semi-automatic segmentation methods.
\end{abstract}

\section{Introduction}

Liver structure analysis from abdominal CTA scans is a key task in many clinical applications. The analysis includes liver contour and volume estimation, blood vessels identification, and, when present, tumor detection and characterization. Clinical applications include hepatomegaly and liver cirrhosis assessment, hepatic volumetry, hepatic transplantation planning, liver regeneration after hepatectomy, evaluation and planning for resection liver surgery, and monitoring of liver metastases, among many others. To be of practical clinical use, the liver analysis must be accurate, robust, fast, and nearly automatic, so that the radiologist can perform it routinely without the assistance of a technician.

Nearly automatic CT-based liver analysis is known to be a very challenging task. The main difficulties include the ambiguity of the liver boundary, the complexity of the liver surface, the presence of surrounding organs, the contrast variability between liver parenchyma and vessels, the different tumor sizes and shapes, and the presence of many small metastases. In addition, liver analysis often requires the simultaneous identification and visualization of liver contours, blood vessels, and tumors, each with their own features and characteristics.

D. Metaxas et al. (Eds.): MICCAI 2008, Part I, LNCS 5241, pp. 85 92, 2008.

(C) Springer-Verlag Berlin Heidelberg 2008 
Over the past decade, researchers have developed a variety of methods for semi-automatic and automatic segmentation and visualization of liver structures.

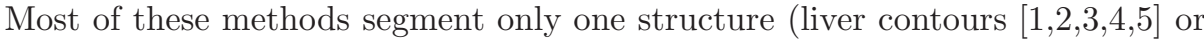
vessels [6]), or segment one structure at a time, usually starting with the liver surface, followed by the vessels and the metastatic lesions. The individual structure segmentation uses various techniques, such as intensity thresholding, region growing, and level-sets based methods. For example, 78 use adaptive binary thresholding to separately segment the liver surface, vessels, and tumors, followed by a deformable model refinement for each. Since it does not use voxel neighborhood information, it may yield noisy or erroneous liver surface segmentations, especially when large tumors are present, as they bias the intensity distribution function. Peitgen et al. 9] describe an edge-based segmentation method for the liver contour and an interactive region-growing method for the vessels and tumors. Since it requires many seeds per CT slice, it is of limited clinical use.

A key observation is that by considering each liver structure individually, the intrinsic relations between the liver parenchyma, vessels, and tumors are lost. This makes the classification more sensitive and error-prone. As an alternative, we propose a simultaneous multi-class segmentation method.

In this paper, we present a new method for the simultaneous segmentation of liver contours, vessels, and metastatic lesions from abdominal CTA scans. The method repeatedly applies multi-resolution, multi-class smoothed Bayesian classification followed by morphological adjustment and active contours refinement. The method requires only one or two seeds (in case of presence of large tumors) for initialization, with no manual adjustment of internal parameters. By using the multi-class and voxel neighborhood information, it significantly improves the discrimination quality of the intensity distribution function for each class. The multi-resolution iterative approach allows the segmentation of the entire liver surface without prior shape information and/or significant user interaction.

The main contribution of the proposed method is the integration of both intensity-based statistical method with geometrical active contours method, into an iterative, multi-scale framework which produced a nearly automatic method for the fully detailed analysis of the liver. Our method yield an accurate and robust results on two clinically validated datasets totaling 56 CTA studies, and achieved a very high score compared to other semi-automatic methods presented in the MICCAI grand-challenge [10.

\section{Method}

Our method consists four steps: 1) multi-class intensity model generation; 2) smoothed Bayesian classification; 3) adaptive morphological adjustment, and; 4) geodesic active contours refinement. The steps are performed in sequence and repeatedly applied to the image until no further changes occur. After each iteration, the internal parameters of the multi-class intensity model are updated before they are used in the smoothed Bayesian classification step. This coupling is designed to overcome a biased classification due to ambiguous liver boundaries 


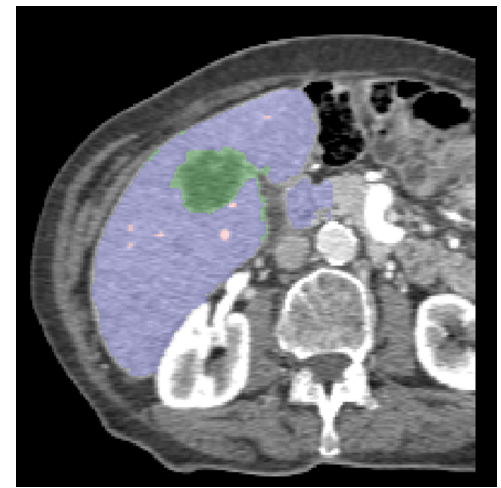

(a) Axial view

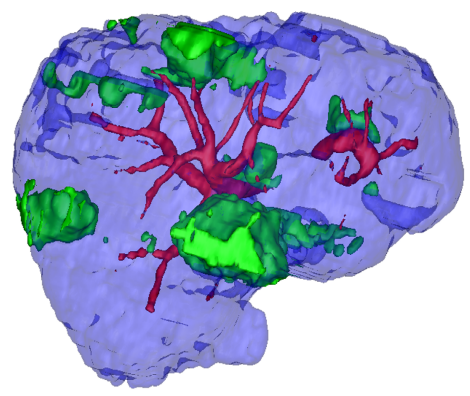

(c) $3 \mathrm{D}$ view

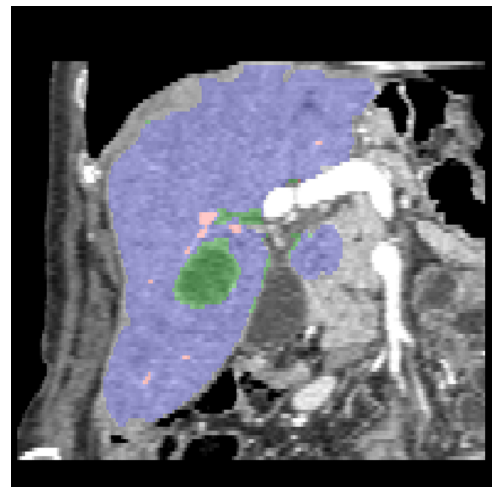

(b) Sagittal view

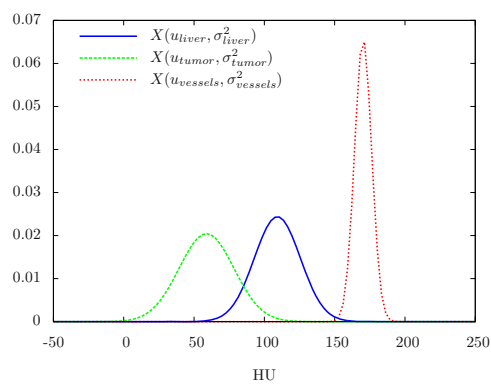

(d) Classes distributions

Fig. 1. Example of a segmented liver dataset. (a)-(b) $2 \mathrm{D}$ views of the segmentation: liver (blue), vessels (red), and tumors (green). (c) 3D visualization. (d) Gaussian distributions of the liver, tumor and vessels classes. The horizontal axis shows Hounsfield unit values; the vertical axis shows the class probabilities.

and biased seed selection. The classification updates the classes models by first computing the mean and variance of the liver and tumor classes from the current liver and tumor regions, and then updates the other classes by computing their mean and variance parameters. The morphological adjustment and the active contours refinement are then applied on the resulting classes to find the new liver and tumors regions. The iterations are necessary to fine-tune the intensity model to improve the classification and to minimize the influence of the initial voxel seed selection. Fig. 1(a) 1(c) illustrates the results of our multi-class segmentation.

To speed up the segmentation and make it more robust and accurate, we use a multi-resolution approach. The first few iterations are performed on a downsampled CTA dataset to obtain a rough contour segmentation. Subsequent iterations are performed on the original CTA dataset until no further improvements can be made. We describe each step in detail next. 


\subsection{Multi-class Intensity Model Generation}

The first step is the construction of an intensity model that differentiates between the liver parenchyma, the liver inner vessels, the metastatic lesions, and the remaining organs and tissues, which are interpreted as background. The main challenges are the ambiguous boundary between the liver parenchyma and the outside organs (e.g the kidney), the ambiguous boundary between liver parenchyma and the liver vessels, and the similarity between lesions intensity values and other background values.

To overcome these difficulties, we first classify the voxels into two main classes, liver and lesions, according to their graylevel value. A refined five-class model is then built for each main class. The central class is the main class (e.g. liver or tumor); the remaining four classes represent other organs and tissues. We model each class with a normal distribution defined by its mean intensity value and variance. In the first iteration, the mean and the variance of a rectangular neighborhood around one manually selected seed inside the liver and, optionally, an additional seed inside a tumor (when the tumor is large) are computed and interpreted as the parameters of the liver and tumor classes. In subsequent iterations, the segmented region from the previous iteration is used to compute the mean and the variance of the liver and tumor classes. The remaining four classes model organs near and far (above/below) the liver and tumor values.

Formally, the liver and tumor classes are defined as: $X_{\text {liver }}, X_{\text {tumor }}$ and the other four classes, $X_{i}$ are modeled as:

$$
X_{\text {central }} \sim N\left(\mu_{\text {central }}, \sigma_{\text {central }}^{2}\right) \quad X_{i} \sim N\left(\mu_{i}, \sigma_{i}^{2}\right)
$$

for each $i, i \in\{$ near - low, near - high, far - low, far - high $\}$, where central is the liver or tumor class. The means of these classes defined as:

$$
\begin{aligned}
\mu_{\text {near-high }} & =\mu_{\text {central }}+k_{\text {near }} \times \sigma_{\text {central }} \\
\mu_{\text {near-low }} & =\mu_{\text {central }}-k_{\text {near }} \times \sigma_{\text {central }} \\
\mu_{\text {far-high }} & =\mu_{\text {central }}+k_{\text {far }} \times \sigma_{\text {central }} \\
\mu_{\text {far-low }} & =\mu_{\text {central }}-k_{\text {far }} \times \sigma_{\text {central }}
\end{aligned}
$$

where the factors $k_{n e a r}$ and $k_{f a r}$ are determined from Chebyshev's inequality:

$$
\left.\operatorname{Pr}\left(\left|X_{i}-\mu_{\text {central }}\right|\right) \geq k \sigma_{\text {central }}\right) \leq \frac{1}{k^{2}}
$$

This inequality ensures that at least $\left(1-\frac{1}{k^{2}}\right) \times 100 \%$ of the values are within $k$ standard deviations from the mean. By setting $k_{n e a r}=2.2$ and $k_{f a r}=4$, we ensure that at least $80 \%$ of the main class voxels will be classified as belonging to the main class, and at least $70 \%$ of the voxels that belong to the near classes will be classified as near, even if the normal distribution assumption is incorrect. This ensures that each voxel from the ambiguous class boundary has a high probability of being both in the central and near classes. Its final classification is then determined from its relation to neighboring voxels. Initially, we set $\sigma_{i}=\sigma_{\text {central }}$ for all classes. Fig. 1(d) shows an example of the Gaussian model of the liver, tumor, and vessels classes, and their overlapping boundaries. 


\subsection{Voxel Classification}

This step uniquely classifies each voxel according to its intensity value and its neighboring voxels 11. Neighborhood information is important since voxel intensity values are correlated. First, we use Bayes rule to compute the probability $C_{(j, i)}$ of a voxel $V_{j}$ with intensity value $v_{j}$ to belong to class $c_{i}$, where $i \in\{$ central, near - low, near - high, far - low, far - high $\}$ :

$$
\operatorname{Pr}\left(v_{j} \mid c_{i}\right) \propto \operatorname{Pr}\left(c_{i} \mid v_{j}\right) \operatorname{Pr}\left(c_{i}\right)
$$

where $\operatorname{Pr}\left(c_{i} \mid v_{j}\right)$ is obtained from the intensity model (Sec. 2.1). Since $\operatorname{Pr}\left(c_{i}\right)$ is usually unavailable, we use a uniform distribution. The resulting five maps quantify the membership probability of each voxel to each class.

Next, we incorporate the neighborhood information to the classification process by smoothing the probability maps $C_{(j, i)}$ for each class $i$ separately using an anisotropic diffusion equation [12. The anisotropic smoothing process smoothes small peeks in the membership probability maps considered as mis-classifications while preserving sharp edges between different objects.

Finally, we apply the Maximum Posterior (MAP) rule:

$$
C_{(j, f \text { inal })}=\underset{c_{i}}{\operatorname{argmax}} \operatorname{Pr}\left(v_{j} \mid c_{i}\right)
$$

to set the final membership of each voxel to the class with the highest probability that the voxel belongs to it. The binary segmentation image is generated by selecting only the voxels that belong to the central class.

This step is applied twice, once for the liver class, and another for the tumor class. The results are combined by taking into account only the tumor voxels inside the liver. Since the vessels appear as bright regions inside the liver, the estimation of the vessels class is based on the $X_{\text {near-high }}$ and $X_{\text {far-high }}$ distributions for voxels inside the liver.

\subsection{Adaptive Morphological Adjustment}

The third step is the identification of the liver region. The previous classification yields most of the liver region, with additional disconnected regions outside the liver, and holes inside the liver. The regions outside the liver correspond to anatomical structures with intensity values similar to the liver, such as the kidney and the spleen. The holes correspond to small tumors and artery and portal veins inside the liver whose intensity values are distinctly different from the liver intensity values because of the imaging contrast agent. To obtain the correct liver segmentation, the disconnected regions must be eliminated, and the holes inside the liver must be filled. This is done with adaptive morphological operations.

We first remove the disconnected regions outside the liver by identifying the largest connected component in the labeled image. Next, the holes inside the liver are classified as tumor or vessels according to the intensity model in Sec. 2.1. Finally, we adjust the liver boundary with an adaptive morphological opening operator. To overcome the inter-patient variablity of the liver size, the radiuses of the morphological operators are linearly depend on the estimated liver volume. 


\subsection{Active Contours Refinement}

The classification and the morphological adjustment may miss parts of the liver volume. In addition, the liver boundaries may be imprecise in several regions. To correct this, we repeatedly apply a fine-tuning active contours segmentation [13]. The active contours segmentation drives the initial surface according to a feature map generated from the original image. To provide a good feature image to the active contours module, we generate a new image with a windowing function:

$$
I^{\prime}(x)= \begin{cases}\mu_{\text {liver }}+\sigma_{\text {liver }} & \text { if } I(x)>\mu_{\text {liver }}+\sigma_{\text {liver }} \\ I(x) & \text { if } \mu_{\text {liver }}-\sigma_{\text {liver }} \leq I(x) \leq \mu_{\text {liver }}+\sigma_{\text {liver }}, \\ \mu_{\text {liver }}-\sigma_{\text {liver }} & \text { if } I(x)<\mu_{\text {liver }}-\sigma_{\text {liver }}\end{cases}
$$

where $I$ is the original CTA data, $I^{\prime}$ is the new image, and $\mu_{\text {liver }}$ and $\sigma_{\text {liver }}$ are the liver class parameters as computed in the intensity model.

\section{$3 \quad$ Experimental Results}

We performed a retrospective study on two validated clinical datasets totaling 56 CTAs. Our implementation used the ITK software library [14] and the smoothed Bayesian classification module [15]. Computations were performed on an Intel Core2 Quad 2.4 GHz PC with 3GB of memory. Table 1 summarize the results.

In the first study, we compared our algorithm estimation of liver volume to a manual ground truth liver volume estimation. In $26 \mathrm{CTA}$ datasets [16], liver contours were manually segmented in each slice by a dedicated technologist on a Vitrea 2 workstation (Vital Images, Plymouth, MN) to serve as the ground truth. The datasets were also segmented with our method. The liver volume was then computed directly from the segmentations and compared by linear regression analysis. The mean absolute difference between the estimated liver volumes was $5.36 \%$ (std $3.48 \%$ ), which was deemed accurate for clinical use. The correlation was 0.98 , which is very and better than those of other automatic methods on the same database [16. The mean difference between initializations with different seeds using our method was $0.54 \%$ (std $0.76 \%$ ), which indicates that our algorithm is highly robust to seed selection. The mean segmentation time using the manual tracing was 7:24 mins, most of it user time. The mean segmentation time using our method was 6:09 mins, most of it computation time, as only a few seconds are required from the radiologist for seed selection. The computation time was deemed reasonable for clinical use. Since we did not have access to the manual segmentation, we could not compare our segmentation to the gold standard using other measures, as we did in the second study.

In the second study, we used 30 publicly available CTA datasets with groundtruth segmentations [10]. The database is divided into two groups. The first group consists of 20 datasets and is used for training and testing; The second group consists of 10 datasets and is used to evaluate segmentation algorithms. We compared our segmentation results to the ground-truth using five metrics: 
Table 1. Summary of experimental results. The first column indicates the database used. The second column shows the estimated mean (std) volume difference. The third column shows the correlation with the ground-truth manual tracing segmentation. The fourth column shows the mean (std) volume estimation variations for the different seed selections, and the last column shows the average running time of our algorithm.

\begin{tabular}{|l|c|c|c|c|}
\hline Database & $\begin{array}{c}\text { Volume } \\
\text { Diff. \% }\end{array}$ & Correlation & $\begin{array}{c}\text { Variability } \\
\%\end{array}$ & $\begin{array}{c}\text { Running } \\
\text { time (mm:ss) }\end{array}$ \\
\hline \hline 1. DB 1 & $5.36(3.48)$ & 0.98 & $0.54(0.76)$ & $6: 09$ \\
\hline 2. DB 2 training & $2.63(1.64)$ & 0.99 & $0.004(0.005)$ & $6: 07$ \\
\hline 3. DB 2 test & $2.78(2.14)$ & - & - & $6: 21$ \\
\hline
\end{tabular}

1) volumetric overlap; 2) relative absolute volume difference; 3) average symmetric absolute surface distance; 4) symmetric RMS surface distance, and; 5) maximum symmetric absolute surface distance. The metrics were computed for each dataset as described in [10. The averages for 10 test datasets (20 training datasets) were as follows: 1) volumetric overlap error $8.55 \%(7.81 \%) ; 2$ ) absolute volume difference $2.78 \%(2.63 \%) ; 3)$ average symmetric surface distance $1.46 \mathrm{~mm}$ (1.28mm); 4) Root Mean Squares (RMS) symmetric surface distance $2.94 \mathrm{~mm}$ (2.55mm); 5) maximum symmetric surface distance $26.72 \mathrm{~mm}(22.77 \mathrm{~mm})$. The correlation between our volume estimation and the ground truth on the training set was 0.99 (note that since the ground truth is not available for the test set, we could not compute the correlation value for it). In addition, we measured the robustness of our method to three different seeds initializations on the 20 training datasets. The absolute volume difference was $0.004 \%$, which is negligible. These results suggest that our method is both accurate and robust to seeds selections. A total score was computed as in [10] for both training and test datasets. Our method achieved a total score of 71.8 on the training set and of 67.87 on the test set. It is ranked forth of the semi-automatic method. However, all other semi-automatic methods require significantly more user interaction and do not provide vessels and tumors segmentation in addition to the liver segmentation.

\section{Conclusion}

We have presented a new nearly-automatic segmentation method for liver analysis. The main advantage of our method is that it simultaneously segments the liver contour, the blood vessels, and tumors inside the liver with only one or two user-selected seeds. Experimental results from two datasets totaling 56 CTA images show that our method is accurate, efficient, and robust to seed selection when compared to manually generated ground truth segmentation. In the future, we plan develop an integrated software package for the visualization and quantitative analysis of the liver to support diagnosis and surgical planning. Project homepage including movies and code is at: http://www.cs.huji.ac. il/ freiman/LiverSeg 


\section{References}

1. Okada, T., Shimada, R., Sato, Y., Hori, M., Yokota, K., Nakamoto, M., Chen, Y.W., Nakamura, H., Tamura, S.: Automated segmentation of the liver from 3D CT images using probabilistic atlas and multi-level statistical shape model. In: Ayache, N., Ourselin, S., Maeder, A. (eds.) MICCAI 2007, Part I. LNCS, vol. 4791, pp. 86-93. Springer, Heidelberg (2007)

2. Heimann, T., Wolf, I., Meinzer, H.: Active shape models for a fully automated 3D segmentation of the liver - an evaluation on clinical data. In: Larsen, R., Nielsen, M., Sporring, J. (eds.) MICCAI 2006. LNCS, vol. 4191, pp. 41-48. Springer, Heidelberg (2006)

3. Pohle, R., Toennies, K.: Segmentation of medical images using adaptive region growing. SPIE Med. Imaging 4322, 1337-1346 (2001)

4. Schenk, A., Prause, G., Peitgen, H.: Efficient semiautomatic segmentation of 3D objects in medical images. In: Delp, S.L., DiGoia, A.M., Jaramaz, B. (eds.) MICCAI 2000. LNCS, vol. 1935, pp. 186-195. Springer, Heidelberg (2000)

5. Chen, T., Metaxas, D.: A hybrid framework for 3D medical image segmentation. Med. Image Analysis 9(6), 547-565 (2005)

6. Fetita, C., Lucidarme, O., Preteux, F., Grenier, P.: CT hepatic venography: 3D vascular segmentation for preoperative evaluation. In: Duncan, J.S., Gerig, G. (eds.) MICCAI 2005. LNCS, vol. 3750, pp. 830-837. Springer, Heidelberg (2005)

7. Gao, L., Heath, D., Kuszyk, B., Fishman, E.: Automatic liver segmentation technique for 3D visualization of CT data. Radiology 201(2), 359-364 (1996)

8. Soler, L., Delingette, H., Malandain, G., Montagnat, J., Ayache, N., Koehl, C., Dourthe, O., Malassagne, B., Smith, M., Mutter, D., Marescaux, J.: Fully automatic anatomical, pathological, and functional segmentation from CT scans for hepatic surgery. Comp. Aided Surgery 6(3), 131-142 (2001)

9. Bourquain, H., Schenk, A., Link, F., Preim, B., Prause, G., Peitgen, H.: Hepavision2a software assistant for preoperative planning in living related liver transplantation and oncologic liver surgery. In: Proc. of the 16th Conf. on Comp. Assisted Radiology and Surgery (CARS 2002), pp. 341-346 (2002)

10. Ginneken, B., Heimann, T., Styner, M.: 3D segmentation in the clinic: A grand challenge. In: Heimann, T., Styner, M., van Ginneken, B. (eds.) 3D Segmentation in the Clinic: A Grand Challenge, pp. 7-15 (2007), http://www.sliver07.org

11. Teo, P., Sapiro, G., Wandell, B.: Anisotropic smoothing of posterior probabilities. In: Proc. of the 1997 Int. Conf. on Image Processing (ICIP 1997), pp. 675-678 (1997)

12. Perona, P., Malik, J.: Scale-space and edge detection using anisotropic diffusion. IEEE Trans. Patt. Analysis Mach. Intelligence 12(7), 629-639 (1990)

13. Caselles, V., Kimmel, R., Sapiro, G.: Geodesic active contours. Int. J. on Comp. Vision 22(1), 61-97 (1997)

14. Ibanez, L., Schroeder, W., Ng, L., Cates, J.: The ITK Software Guide. Kitware, Inc. ISBN 1-930934-15-7 (2005), http://www.itk.org/ItkSoftwareGuide.pdf

15. Melonakos, J., Krishnan, K., Tannenbaum, A.: An ITK filter for bayesian segmentation: itkbayesianclassifierimagefilter. Insight Journal (2006), http://www.insight-journal.org

16. Sosna, J., Berman, P., Azraq, Y., Libson, E.: Automated liver segmentation and volume calculation from mdct using a bayesian likelihood maximization technique: Comparison with manual tracing technique. In: 92nd Sci. Ass. and Ann. Meet. of the Radiological Society of N.A (RSNA 2006) (2006) 